\title{
Alkylazinylcarbonitriles as building blocks in organic synthesis: synthesis of 3-amino-7-arylhyrazonothieno-7H-[3,4-c]-pyridine- 4,6-diones and pyrido-[3,4-c]-pyridazine-5-carbonitrile
}

\author{
Saleh Mohamed Al-Mousawi ${ }^{a}$ *, Ismail Abdelshafy Abdelhamid ${ }^{\mathrm{b}}$ and \\ Moustafa Sherief Moustafa ${ }^{a}$ \\ ${ }^{a}$ Department of Chemistry, Faculty of Science, University of Kuwait; P.O. Box 5969; Safat; \\ 13060 Kuwait \\ ${ }^{b}$ Department of Chemistry, Faculty of Science, Cairo University, Giza, A. R. \\ E-mail: salehalmousawi@hotmail.com
}

\begin{abstract}
A series of 5-arylhydrazono-1,2,5,6-tetrahydro-1,4-dialkyl-2,6-dioxopyridine-3-carbonitriles 4 has been prepared and reacted with elemental sulfur to yield the thieno[3,4-c]pyridine-4,6-dione 5. Reaction of $\mathbf{5}$ with dimethyl acetylenedicarboxylate afforded arylazoisoquinolines 7. Condensation of 4 with dimethylformamide dimethylacetal afforded pyrido[3,4-c]pyridazine-5carbonitrile 9.
\end{abstract}

\section{Introduction}

The reaction of alkylheteroaromatic carbonitriles with elemental sulfur seems to be an interesting route for the synthesis of condensed aminothiophenes. This methodology has been utilized by Elnagdi et al. ${ }^{1-8}$ and Döpp et al. ${ }^{9,10}$ as precursors to thienopyridazines, thienocoumarines and thienoquinolines, which were subsequently reacted with dienophiles yielding benzofused heteroaromatics. The scope of this approach for the synthesis of phthalazines as well as condensed benzopyrans has been defined by the recent work of Döpp and coworkers as well as Elnagdi et al. (Scheme 1). ${ }^{7,8}$ Another approach also utilized by Elnagdi et al. ${ }^{7}$ to synthesis condensed azines $\mathbf{V}$, is by condensation of $\mathbf{I}$ with dimethylformamide-dimethylacetal and subsequent treatment of the formed enamine IV with primary aromatic amines. 


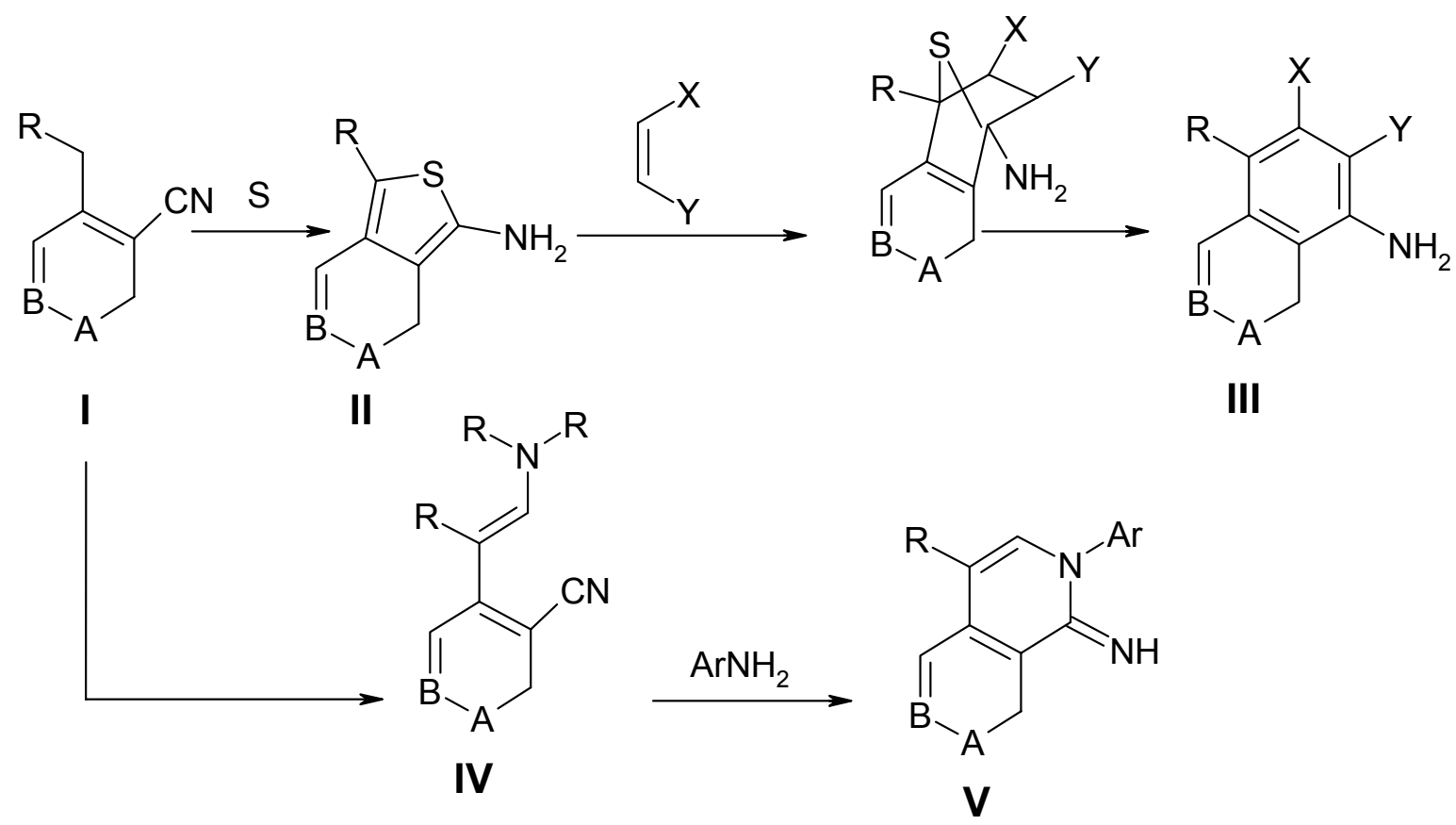

\section{Scheme 1}

It seemed of value to determine if these approaches can be extended to constitute a new general route to aminoarylazoisoquinoline 7 starting from the readily obtainable $\mathbf{3}$. Compound 3 is prepared by reacting a mixture of 3-oxoesters $\mathbf{1}$ with cyanoacetamides $\mathbf{2}$ (which are prepared insitu by reacting ethyl cyanoacetate with primary amines) either in microwave equipment or by reflux. Both methods afforded 3a-c in excellent yields. Synthesis of compounds 3a-c have been reported earlier in the literature by refluxing a mixture of $\mathbf{1}$ and $\mathbf{2}$ in absence of solvent (Scheme 2). ${ }^{11}$ Compounds 3 could be readily coupled with aromatic diazonium salts to yield the corresponding arylazo derivatives 4 a-d (Scheme 2). 
<smiles>[X]CC(=O)CC(=O)O[Z17]</smiles>

$$
\begin{aligned}
& \text { 3a, } X=\mathrm{CH}_{3} ; \mathrm{R}=\mathrm{CH}_{2} \mathrm{Ph} \\
& \text { b, } X=\mathrm{CH}_{3} ; \mathrm{R}=\mathrm{CH}_{2} \mathrm{CH}_{3} \\
& \text { c, } \mathrm{X}=\mathrm{H} \quad ; \mathrm{R}=\mathrm{CH}_{2} \mathrm{Ph}
\end{aligned}
$$<smiles>[X]CC1=C(C#N)C(=O)N([R])C(=O)/C1=N/N[Te]</smiles>
4a, $\mathrm{X}=\mathrm{CH}_{3} ; \mathrm{R}=\mathrm{CH}_{2} \mathrm{Ph} ; \mathrm{Ar}=\mathrm{C}_{6} \mathrm{H}_{5}$
b, $\mathrm{X}=\mathrm{CH}_{3} ; \mathrm{R}=\mathrm{CH}_{2}-\mathrm{CH}_{3} ; \mathrm{Ar}=\mathrm{C}_{6} \mathrm{H}_{4} \mathrm{OCH}_{3}$.(p)
c, $\mathrm{X}=\mathrm{CH}_{3} ; \mathrm{R}=\mathrm{CH}_{2}-\mathrm{CH}_{3} ; \mathrm{Ar}=\mathrm{C}_{6} \mathrm{H}_{5}$
$\mathrm{d}, \mathrm{X}=\mathrm{H} \quad ; \mathrm{R}=\mathrm{CH}_{2}-\mathrm{Ph} ; \mathrm{Ar}=\mathrm{C}_{6} \mathrm{H}_{5}$

\section{Scheme 2}

Compounds 4a-d reacted smoothly with sulfur either in microwave equipment or via reflux in dioxan in the presence of piperidine to afford the thienopyridines 5a-d in good yields (Scheme 3).<smiles>[X]CC1=C(C#N)C(=O)N([R])C(=O)C1=NN[Ga]</smiles>

4

5a, $\mathrm{X}=\mathrm{CH}_{3} ; \mathrm{R}=\mathrm{CH}_{2} \mathrm{Ph} ; \mathrm{Ar}=\mathrm{C}_{6} \mathrm{H}_{5}$

b, $\mathrm{X}=\mathrm{CH}_{3} ; \mathrm{R}=\mathrm{CH}_{2}-\mathrm{CH}_{3} ; \mathrm{Ar}=\mathrm{C}_{6} \mathrm{H}_{4} \mathrm{OCH}_{3}-$

c, $\mathrm{X}=\mathrm{CH}_{3} ; \mathrm{R}=\mathrm{CH}_{2}-\mathrm{CH}_{3} ; \mathrm{Ar}=\mathrm{C}_{6} \mathrm{H}_{5}$

$\mathrm{d}, \mathrm{X}=\mathrm{H} \quad ; \mathrm{R}=\mathrm{CH}_{2}-\mathrm{Ph} ; \mathrm{Ar}=\mathrm{C}_{6} \mathrm{H}_{5}$

\section{Scheme 3}

Reaction of 5d with dimethyl acetylenedicarboxylate afforded arylazoisoquinoline 7 (Scheme 4). Attempts to add other dienophiles resulted in the formation of 4. 


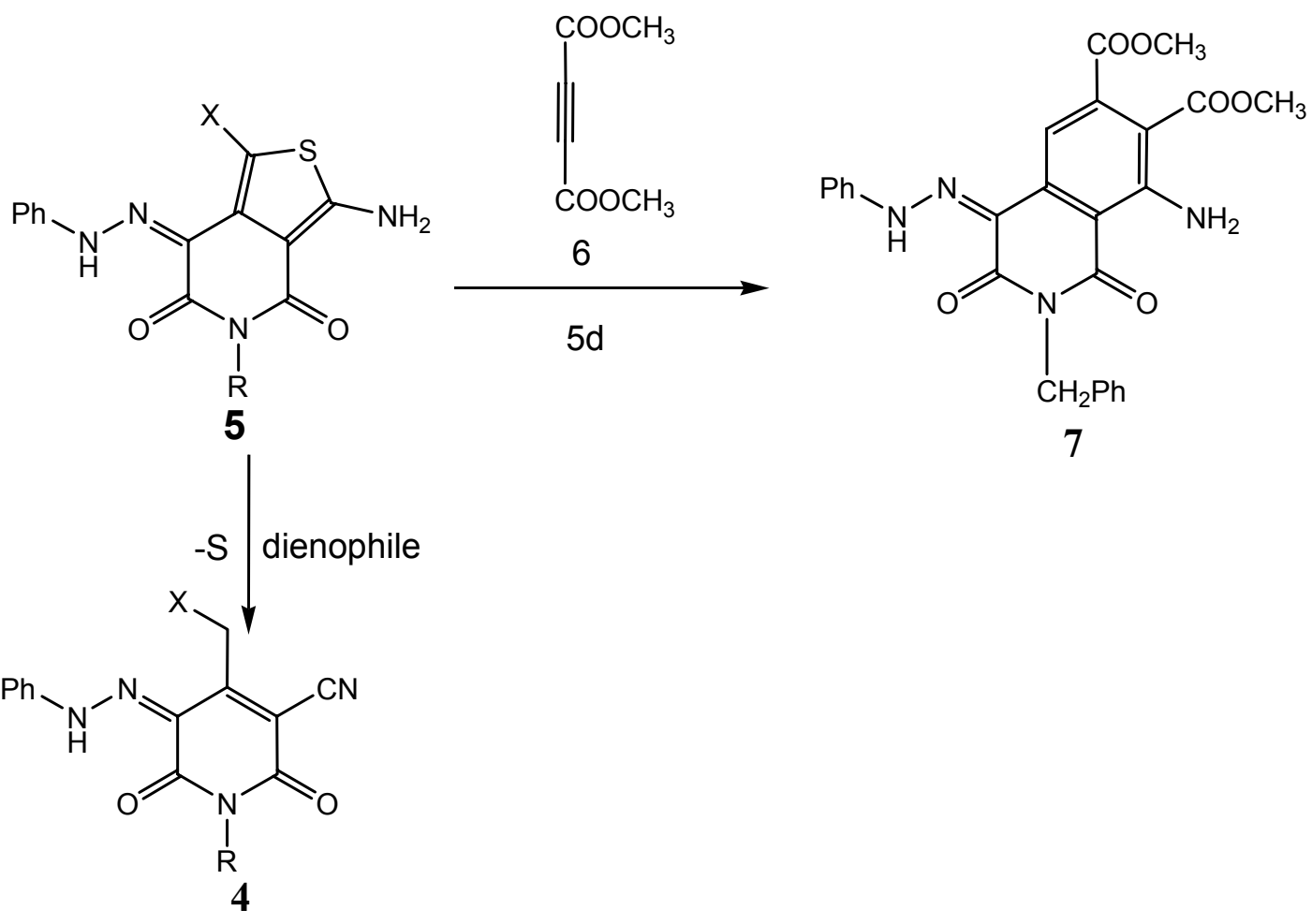

\section{Scheme 4}

Compound 4d readily condensed with dimethylformamide dimethylacetal (DMFDMA) to yield the pyrido[3,4-c]pyridazine-5-carbonitrile 9 most likely via intermediacy of $\mathbf{8}$ (Scheme 5).

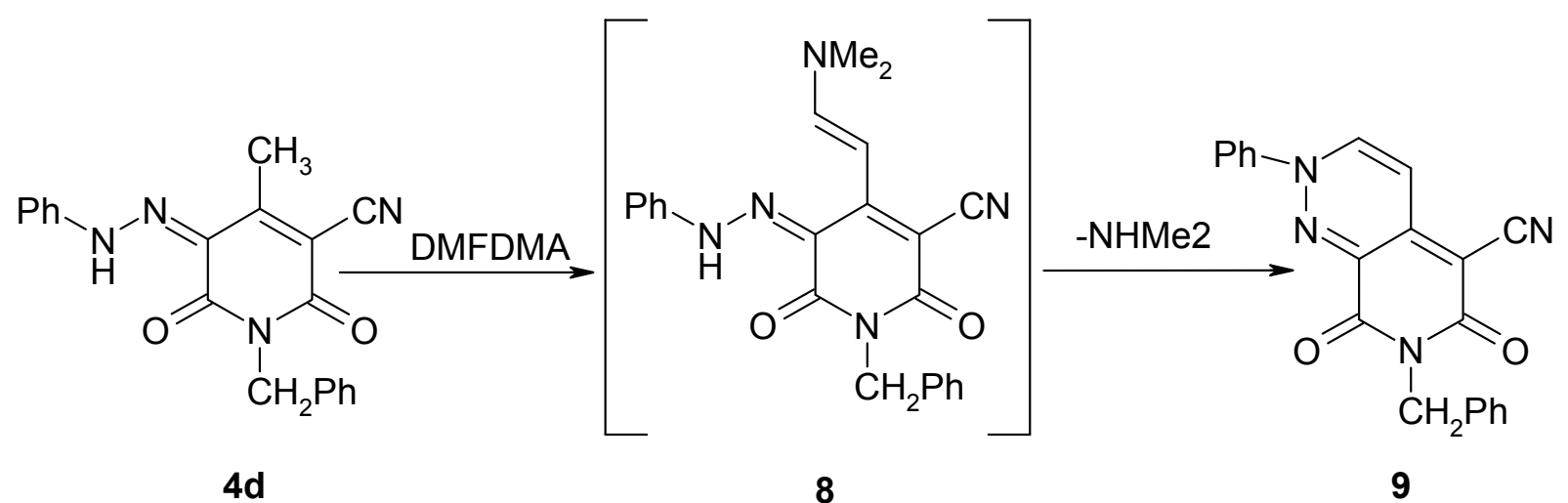

\section{Scheme 5}




\section{Experimental Section}

General Procedures. All melting points are uncorrected and determined on a Sanyo (Gallaenkamp) instrument. Infrared spectra were recorded in $\mathrm{KBr}$ and determined on a PerkinElmer 2000 FT-IR system. ${ }^{1} \mathrm{H}$ NMR and ${ }^{13} \mathrm{C}$ NMR spectra were determined on a Bruker DPX $400 \mathrm{MHz}$ spectrometer in $\mathrm{CDCl}_{3}$ or DMSO- $\mathrm{d}_{6}$ as solvent and TMS as internal standard; chemical shifts are reported in $\delta(\mathrm{ppm})$. Mass spectra were measured on MS 30 and MS 9 (AEI) spectrometers, with EI $70 \mathrm{EV}$. Elemental analyses were measured by means of a LEOCHNS-932 Elemental Analyzer.

1-Benzyl-4-ethyl-2,6-dioxo-1,2,5,6-tetrahydropyridin-3-carbonitrile (3a). A mixture of ethyl cyanoacetate $(1.13 \mathrm{~g}, 1 \mathrm{mmol})$ and the benzylamine $(1.07 \mathrm{~g}, 1 \mathrm{mmol})$ was stirred until a clear solution was obtained, and then methyl propionoylacetate $(1.30 \mathrm{~g}, 1 \mathrm{mmol})$ was added. The mixture was refluxed for 6 hours. The solution was diluted with water and acidified with hydrochloric acid to give a white product, yield $2.26 \mathrm{~g}(89 \%), \mathrm{mp} .229-31{ }^{\circ} \mathrm{C}$. Anal. Calcd. for $\mathrm{C}_{15} \mathrm{H}_{14} \mathrm{~N}_{2} \mathrm{O}_{2}$ (254.29): C, 70.85; H, 5.55; N, 11.02. Found: C, 70.29; H, 5.41; N, 11.26. IR (KBr, $\mathrm{cm}^{-1}$ ): $3447(\mathrm{OH}), 2216(\mathrm{CN}), 1653(\mathrm{CO}) ;{ }^{1} \mathrm{H}$ NMR (400 MHz, DMSO-d6): $\delta$, ppm 1.09 (t, 3H, $\mathrm{CH}_{3}, \mathrm{~J}=8 \mathrm{~Hz}$ ), 2.41 (q, $2 \mathrm{H}, \mathrm{CH}_{2}, J=8 \mathrm{~Hz}$ ), $5.04\left(\mathrm{~s}, 2 \mathrm{H}, \mathrm{CH}_{2}\right), 5.58$ (s, $1 \mathrm{H}, \mathrm{CH}$ pyridine), 7.217.36 (m, 5H, Ar-H), 10.46 (br, 1H, OH, $\mathrm{D}_{2} \mathrm{O}$ exchangeable); ${ }^{13} \mathrm{C}$ NMR (100 MHz, DMSO-d6): $\delta$, ppm 14.3, 26.3, 28.4, 45.9, 74.3, 87.9, 92.0, 128.4, 129.4, 137.0, 162.0, 163.8, 164.6. MS (EI): $\mathrm{m} / \mathrm{z}(\%)=254\left(\mathrm{M}^{+}\right)$.

1,4-Diethyl-2,6-dioxo-1,2,5,6-tetrahydropyridine-3-carbonitrile (3b). Compound $3 \mathrm{~b}$ was obtained from ethyl cyanoacetate $(1.13 \mathrm{~g}, 1 \mathrm{mmol})$, ethylamine $(0.45 \mathrm{~g}, 1 \mathrm{mmol})$ and methyl propionoylacetate $(1.30 \mathrm{~g}, 1 \mathrm{mmol})$, in a way similar to that described for synthesis of 3a, white powder, yield $1.69 \mathrm{~g} \mathrm{(88 \% ),} \mathrm{mp.} \mathrm{219-20}{ }^{\circ} \mathrm{C}$. Anal. Calcd. for $\mathrm{C}_{10} \mathrm{H}_{12} \mathrm{~N}_{2} \mathrm{O}_{2}$ (192.22): C, 62.49; H, 6.29; N, 14.57. Found: C, 62.59; H, 6.31; N, 14.55. IR (KBr, $\left.\mathrm{cm}^{-1}\right): 3439(\mathrm{OH}), 2222(\mathrm{CN}), 1641$ (CO) $\mathrm{cm}^{-1} ;{ }^{1} \mathrm{H}$ NMR (400 MHz, DMSO-d6): $\delta$, ppm 1.07 (t, $\left.3 \mathrm{H}, \mathrm{CH}_{3}, J=8 \mathrm{~Hz}\right), 1.14$ (t, $3 \mathrm{H}$, $\mathrm{CH}_{3}, J=8 \mathrm{~Hz}$ ), 2.48 (q, $2 \mathrm{H}, \mathrm{CH}_{2}, J=8 \mathrm{~Hz}$ ), 3.91 (q, $\left.2 \mathrm{H}, \mathrm{CH}_{2}-\mathrm{N}, J=8 \mathrm{~Hz}\right), 5.65$ (s, $1 \mathrm{H}, \mathrm{CH}$ pyridine), 7.00 (br, $1 \mathrm{H}, \mathrm{OH}, \mathrm{D}_{2} \mathrm{O}$ exchangeable); ${ }^{13} \mathrm{C}$ NMR (100 MHz, DMSO-d6): $\delta$, ppm 12.0, 14.3, 28.3, 36.9, 88.7, 91.4, 118.2, 161.4, 161.6, 164.5. MS (EI): $\mathrm{m} / \mathrm{z}(\%)=192\left(\mathrm{M}^{+}\right)$.

1-Benzyl-4-methyl-2,6-dioxo-1,2,5,6-tetrahydropyridin-3-carbonitrile (3c). Compound 3c was obtained from ethyl cyanoacetate $(1.13 \mathrm{~g}, 1 \mathrm{mmol})$, benzylamine $(1.07 \mathrm{~g}, 1 \mathrm{mmol})$ and ethyl acetoacetate $(1.30 \mathrm{~g}, 1 \mathrm{mmol})$, in a way similar to that described for synthesis of compound 3a, white powder, yield 2.16 g $(90 \%)$, mp. $250-52{ }^{\circ} \mathrm{C}$. Anal.Calcd. for $\mathrm{C}_{14} \mathrm{H}_{12} \mathrm{~N}_{2} \mathrm{O}_{2}$ (240.26): C, 69.99; H, 5.03; N, 11.66. Found: C, 69.65; H, 5.04; N, 11.78. IR (KBr, cm $\left.{ }^{-1}\right): 2218(\mathrm{CN}), 1648$ (CO); ${ }^{1} \mathrm{H}$ NMR (400 MHz, DMSO-d6): $\delta$, ppm 2.14 (s, 3H, $\mathrm{CH}_{3}$ ), $5.02\left(\mathrm{~s}, 2 \mathrm{H}, \mathrm{CH}_{2}\right), 5.69$ (s, $1 \mathrm{H}$, $\mathrm{CH}$ pyridine), 7.17-7.38 (m, 5H, Ar-H), 9.98 (br, 1H, OH, $\mathrm{D}_{2} \mathrm{O}$ exchangeable); ${ }^{13} \mathrm{C}$ NMR (100 MHz, DMSO-d6): $\delta$, ppm 11.9, 21.6, 42.2, 44.6, 93.2, 118.4, 128.4, 129.5, 159.7, 161.2, 163.2. MS (EI): $\mathrm{m} / \mathrm{z}(\%)=240\left(\mathrm{M}^{+}\right)$. 


\section{1-Benzyl-4-ethyl-2,6-dioxo-5-(phenylhydrazono)-1,2,5,6-tetrahydropyridine-3-carbonitrile}

(4a). A cold solution of aryldiazonium salt $(10 \mathrm{mmol})$ was prepared by adding a solution of sodium nitrite $\left(0.7 \mathrm{~g}\right.$ into $\left.10 \mathrm{~mL} \mathrm{H}_{2} \mathrm{O}\right)$ to a cold solution of arylamine hydrochloride $(10 \mathrm{mmol}$ of arylamine in $5 \mathrm{~mL}$ concentrated $\mathrm{HC} 1)$ with stirring. The resulting solution of aryldiazonium chloride was then added to a cold solution of pyridones $\mathbf{3 a}(2.54 \mathrm{~g}, 10 \mathrm{mmol})$ in ethanol $(50 \mathrm{~mL})$ containing sodium acetate ( $2 \mathrm{~g}$ dissolved in $5 \mathrm{ml}$ of water). The reaction mixture was stirred for 1 hr. The solid product, so formed, was collected by filtration and crystallized from dioxan to afford yellow crystals, yield $3.29 \mathrm{~g}(92 \%), \mathrm{mp} .222-23{ }^{\circ} \mathrm{C}$. Anal. Calcd. for $\mathrm{C}_{21} \mathrm{H}_{18} \mathrm{~N}_{4} \mathrm{O}_{2}(358.40)$ : C, 70.38; H, 5.06; N, 15.63. Found: C, 69.99; H, 4.91; N, 15.73. IR ( $\left.\mathrm{KBr}, \mathrm{cm}^{-1}\right): 3420(\mathrm{NH})$, 2221 (CN), 1671, 1641 (2CO); ${ }^{1} \mathrm{H}$ NMR (400 MHz, DMSO-d6): $\delta$, ppm 1.27 (t, 3H, $\mathrm{CH}_{3}, J=$ $7.6 \mathrm{~Hz}$ ), 2.96 (q, 2H, $\mathrm{CH}_{2}, J=7.6 \mathrm{~Hz}$ ), 5.05 (s, 2H, $\left.\mathrm{CH}_{2}\right), 7.25-7.71$ (m, 10H, Ar H), 14.56 (s, $1 \mathrm{H}, \mathrm{NH}, \mathrm{D}_{2} \mathrm{O}$ exchangeable); ${ }^{13} \mathrm{C}$ NMR (100 MHz, DMSO-d6): $\delta$, ppm 15.1, 24.8, 43.5, 115.9, $118.5,122.6,128.2,128.5,128.7,129.4,130.9,137.3,142.1,161.5,161.6,165.7 . \mathrm{MS}(\mathrm{EI}): \mathrm{m} / \mathrm{z}$ $(\%)=358\left(\mathrm{M}^{+}\right)$.

\section{1,4-Diethyl-5-[(4-methoxyphenyl)-hydrazono]-2,6-dioxo-1,2,5,6-tetrahydropyridine-3-}

carbonitrile (4b). Compound 4b was obtained from compound 3b (1.92 g, $10 \mathrm{mmol}$ ) and aryldiazonium chloride $(10 \mathrm{mmol})$, in a way similar to that described for synthesis of $\mathbf{4 a}$, yellow crystals, yield $3.00 \mathrm{~g}(92 \%)$, mp. 226-28 ${ }^{\circ} \mathrm{C}$. Anal. Calcd. for $\mathrm{C}_{17} \mathrm{H}_{18} \mathrm{~N}_{4} \mathrm{O}_{3}$ (326.36): C, 62.57; H, 5.56; N, 17.17. Found: C, 62.39; H, 5.58; N, 17.18. IR (KBr, cm $\left.{ }^{-1}\right): 3440(\mathrm{NH}), 2225(\mathrm{CN})$, 1676, 1645 (2CO); ${ }^{1} \mathrm{H}$ NMR (400 MHz, $\left.\mathrm{CDCl}_{3}\right)$ : $\delta$, ppm 1.25 (t, 3H, $\left.\mathrm{CH}_{3}, J=7.2 \mathrm{~Hz}\right), 1.35$ (t, $\left.3 \mathrm{H}, \mathrm{CH}_{3}, J=7.6 \mathrm{~Hz}\right), 3.03$ (q, 2H, $\left.\mathrm{CH}_{2}, J=7.6 \mathrm{~Hz}\right), 3.88\left(\mathrm{~s}, 3 \mathrm{H}, \mathrm{OCH}_{3}\right), 4.04$ (q, $2 \mathrm{H}, \mathrm{CH}_{2}, J=$ $7.2 \mathrm{~Hz}), 7.01$ (d, 2H, p-tolyl-H, $J=8 \mathrm{~Hz}), 7.45$ (d, 2H, p-tolyl H, $J=8 \mathrm{~Hz}) ;{ }^{13} \mathrm{C}$ NMR $(100$ $\left.\mathrm{MHz}, \mathrm{CDCl}_{3}\right)$ : $\delta$, ppm 13.5, 15.0, 24.6, 35.6, 56.2, 100.0, 115.1, 115.8, 119.2, 121.5, 134.8, 159.9, 160.8, 162.4, 164.4. MS (EI): $\mathrm{m} / \mathrm{z}(\%)=326\left(\mathrm{M}^{+}\right)$.

\section{1,4-Diethyl-2,6-dioxo-5-(phenylhydrazono)-1,2,5,6-tetrahydropyridine-3-carbonitrile (4c).}

Compound 4c was obtained from compound 3c (2.4 g, $10 \mathrm{mmol})$ and aryldiazonium chloride (10 $\mathrm{mmol})$, in a way similar to that described for synthesis of 4a, orange crystals, yield $2.6 \mathrm{~g}(88 \%)$, mp. 207-09 ${ }^{\circ} \mathrm{C}$. Anal. Calcd. for $\mathrm{C}_{16} \mathrm{H}_{16} \mathrm{~N}_{4} \mathrm{O}_{2}$ (296.33): C, 64.85; H, 5.44; N, 18.91. Found: C, 64.69; H, 5.53; N, 18.93. IR (KBr, cm $\left.{ }^{-1}\right): 3434(\mathrm{NH}), 2222(\mathrm{CN}), 1672,1641$ ( $\left.2 \mathrm{CO}\right) ;{ }^{1} \mathrm{H}$ NMR $\left(400 \mathrm{MHz}, \mathrm{CDCl}_{3}\right): \delta$, ppm $1.25\left(\mathrm{t}, 3 \mathrm{H}, \mathrm{CH}_{3}, J=7.2 \mathrm{~Hz}\right), 1.37$ ( t, 3H, $\left.\mathrm{CH}_{3}, J=7.6 \mathrm{~Hz}\right), 3.05$ (q, $\left.2 \mathrm{H}, \mathrm{CH}_{2}, J=7.6 \mathrm{~Hz}\right), 4.04\left(\mathrm{q}, 2 \mathrm{H}, \mathrm{CH}_{2}, J=7.2 \mathrm{~Hz}\right), 7.28-7.51(\mathrm{~m}, 5 \mathrm{H}, \mathrm{Ar} \mathrm{H}), 15.11(\mathrm{~s}, 1 \mathrm{H}, \mathrm{NH}$, $\mathrm{D}_{2} \mathrm{O}$ exchangeable). $\mathrm{MS}(\mathrm{EI}): \mathrm{m} / \mathrm{z}(\%)=296\left(\mathrm{M}^{+}\right)$.

\section{1-Benzyl-4-methyl-2,6-dioxo-5-(phenylhydrazono)-1,2,5,6-tetrahydropyridine-3-}

carbonitrile (4d). Compound $\mathbf{4 d}$ was obtained from compound $\mathbf{3 d}(10 \mathrm{mmol})$ and aryldiazonium chloride (10 $\mathrm{mmol})$, in a way similar to that described for synthesis of $\mathbf{4 a}$, yellow crystals, yield $3.1 \mathrm{~g}(90 \%)$, mp. $239-40{ }^{\circ} \mathrm{C}$. Anal. Calcd. for $\mathrm{C}_{20} \mathrm{H}_{16} \mathrm{~N}_{4} \mathrm{O}_{2}$ (344.38): C, 69.76; H, 4.68; N, 16.27. Found: C, 69.81; H, 4.82; N, 16.14. IR (KBr, cm $\left.{ }^{-1}\right): 3437(\mathrm{NH}), 2222(\mathrm{CN}), 1673,1647$ (2CO); ${ }^{1} \mathrm{H}$ NMR (400 MHz, $\left.\mathrm{CDCl}_{3}\right): \delta$, ppm $2.62\left(\mathrm{~s}, 3 \mathrm{H}, \mathrm{CH}_{3}\right), 5.18\left(\mathrm{~s}, 2 \mathrm{H}, \mathrm{CH}_{2}\right), 7.30-7.52(\mathrm{~m}, 10 \mathrm{H}, \mathrm{Ar}$ $\mathrm{H}), 15.00$ (s, 1H, NH, $\mathrm{D}_{2} \mathrm{O}$ exchangeable). $\mathrm{MS}(\mathrm{EI}): \mathrm{m} / \mathrm{z}(\%)=344\left(\mathrm{M}^{+}\right)$.

3-Amino-5-benzyl-1-methyl-7-(phenylhydrazono)-7H-thieno[3,4-c]pyridine-4,6-dione (5a) 
Method A. To a suspension of compounds $4 \mathbf{a}(3.58 \mathrm{~g}, 10 \mathrm{mmol})$ in dioxan $(2 \mathrm{ml})$, elemental sulphur $(0.32 \mathrm{~g}, 10 \mathrm{mmol})$ and few drops of piperidine were added. The reaction mixture was refluxed for 8 hours and then poured onto water. The solid product, so formed, was collected by filtration and crystallized from ethanol as red crystals.

Method B. To a suspension of compounds $4 \mathbf{a}(3.58 \mathrm{~g}, 10 \mathrm{mmol})$ in dioxan $(2 \mathrm{ml})$, elemental sulphur $(0.32 \mathrm{~g}, 10 \mathrm{mmol})$ and few drops of piperidine were added. The reaction mixture was irradiated in focused microwave at $150 \mathrm{Watt}, 200{ }^{\circ} \mathrm{C}$ for 5 minutes and then poured onto water. The solid product, so formed, was collected by filtration and crystallized from dioxin, red crystals; yield $3.12 \mathrm{~g}(80 \%)$, mp. 80-82 ${ }^{\circ} \mathrm{C}$. Anal. Calcd. for $\mathrm{C}_{21} \mathrm{H}_{18} \mathrm{~N}_{4} \mathrm{O}_{2} \mathrm{~S}$ (390.47): C, 64.60; $\mathrm{H}$, 4.65; N, 14.35. Found: C, 64.62; H, 4.60; N, 14.57. IR (KBr, $\left.\mathrm{cm}^{-1}\right): 3453$ and $3439\left(\mathrm{NH}_{2}\right) ; 3317$ $(\mathrm{NH}) ; 1659 ; 1679$ (2CO); ${ }^{1} \mathrm{H}$ NMR (400 MHz, $\left.\mathrm{CDCl}_{3}\right): \delta$, ppm 2.5 (s, 3H, $\left.\mathrm{CH}_{3}\right), 5.05$ (s, 2H, $\mathrm{CH}_{2}$ ), 7.03-7.38 (m, 10H, Ar-H), 7.71 (s, 2H, NH${ }_{2}, \mathrm{D}_{2} \mathrm{O}$ exchangeable), 13.49 (s, 1H, NH, $\mathrm{D}_{2} \mathrm{O}$ exchangeable); ${ }^{13} \mathrm{C}$ NMR (100 $\left.\mathrm{MHz}, \mathrm{CDCl}_{3}\right): \delta$, ppm 16.3, 43.7, 114.9, 115.2, 123.3, 123.6, 125.0, 127.9, 128.8, 129.0, 130.0, 137.9, 143.3, 160.1, 162.1, 164. MS (EI): m/z (\%) = $390\left(\mathrm{M}^{+}\right)$.

3-Amino-5-ethyl-7-[(4-methoxyphenyl)hydrazono)]-1-methyl-7H-thieno[3,4-c]pyridine-4,6dione (5b). Compound 5b was prepared from compound 4b (3.26 g, $10 \mathrm{mmol})$ and elemental sulphur $(0.32 \mathrm{~g}, 10 \mathrm{mmol})$, in a way similar to that described for synthesis of compound $\mathbf{5 a}$, red crystals; yield $3.04 \mathrm{~g}$ (85\%), mp. $245-46{ }^{\circ} \mathrm{C}$. Anal. Calcd. for $\mathrm{C}_{17} \mathrm{H}_{18} \mathrm{~N}_{4} \mathrm{O}_{3} \mathrm{~S}$ (358.42): C, 56.97; H, 5.06; N, 15.63. Found: C, 56.93; H, 5.06; N, 15.75. IR (KBr, cm $\left.{ }^{-1}\right): 3414$ and $3291\left(\mathrm{NH}_{2}\right)$, 3165 (NH), 1648, 1611 (2 CO); ${ }^{1} \mathrm{H}$ NMR (400 MHz, DMSO-d6): $\delta$, ppm 1.10 (t, 3H, $\mathrm{CH}_{3}, J=$ $7.2 \mathrm{~Hz}$ ), 2.50 (s, 3H, $\left.\mathrm{CH}_{3}\right), 3.39$ (s, 3H, $\left.\mathrm{OCH}_{3}\right), 3.85$ (q, 2H, $\left.\mathrm{CH}_{2}, J=7.2 \mathrm{~Hz}\right), 6.97$ (d, 2H, ptolyl-H, $J=9.2 \mathrm{~Hz}$ ), 7.32 (d, 2H, p-tolyl-H, $J=9.2 \mathrm{~Hz}$ ), 7.62 (s, 2H, $\mathrm{NH}_{2}, \mathrm{D}_{2} \mathrm{O}$ exchangeable), 13.61 (s, 1H, NH, $\mathrm{D}_{2} \mathrm{O}$ exchangeable); ${ }^{13} \mathrm{C}$ NMR (100 MHz, DMSO-d6): $\delta$, ppm 14.3, 16.4 , $34.1,56.3,102.4,112.2,115.8,116.6,123,124.9,137.5,156.4,161.3,161.7,163.6$. MS (EI): $\mathrm{m} / \mathrm{z}(\%)=358\left(\mathrm{M}^{+}\right)$.

3-Amino-5-ethyl-1-methyl-7-(phenylhydrazono)-7H-thieno[3,4-c]pyridine-4,6-dione (5c).

Compound 5c was prepared from compound $4 \mathbf{c}(2.96 \mathrm{~g}, 10 \mathrm{mmol})$ and elemental sulphur $(0.32$ $\mathrm{g}, 10 \mathrm{mmol}$ ), in a way similar to that described for synthesis of 5a, red crystals, yield $2.62 \mathrm{~g}$ (80\%), mp. 241-43 ${ }^{\circ}$ C. Anal. Calcd. for $\mathrm{C}_{16} \mathrm{H}_{16} \mathrm{~N}_{4} \mathrm{O}_{2} \mathrm{~S}$ (328.40): C, 58.52; H, 4.91; N, 17.06 . Found: C, 58.36; H, 4.80; N, 17.08. IR $\left(\mathrm{KBr}, \mathrm{cm}^{-1}\right): 3406$ and $3281\left(\mathrm{NH}_{2}\right), 3167(\mathrm{NH}), 1654$, 1651 (2 CO); ${ }^{1} \mathrm{H}$ NMR (400 MHz, DMSO-d6): $\delta$, ppm 1.11 (t, 3H, $\mathrm{CH}_{3}, J=8 \mathrm{~Hz}$ ), 2.56 (s, 3H, $\left.\mathrm{CH}_{3}\right), 3.05\left(\mathrm{q}, 2 \mathrm{H}, \mathrm{CH}_{2}, J=8 \mathrm{~Hz}\right), 7.02-7.49(\mathrm{~m}, 5 \mathrm{H}, \mathrm{Ar}-\mathrm{H}), 7.66\left(\mathrm{~s}, 2 \mathrm{H}, \mathrm{NH}_{2}, \mathrm{D}_{2} \mathrm{O}\right.$ exchangeable), $13.58\left(\mathrm{~s}, 1 \mathrm{H}, \mathrm{NH}, \mathrm{D}_{2} \mathrm{O}\right.$ exchangeable) $;{ }^{13} \mathrm{C}$ NMR (100 MHz, DMSO-d6): $\delta$, ppm 14.2, 16.5, 34.2, 102.3, 113.5, 115.3 123.7, 124, 124.7, 130.5, 143.8, 161.2, 161.8, 163.6. MS (EI): $\mathrm{m} / \mathrm{z}(\%)=328\left(\mathrm{M}^{+}\right)$.

3-Amino-5-benzyl-7-(phenylhydrazono)-7H-thieno[3,4-c]pyridine-4,6-dione(5d). Compound 5d was prepared from compound $4 \mathbf{d}(3.44 \mathrm{~g}, 10 \mathrm{mmol})$ and elemental sulphur $(0.32 \mathrm{~g}, 10$ $\mathrm{mmol})$, in a way similar to that described for synthesis of $\mathbf{5 a}$, red crystals, yield $2.93 \mathrm{~g}(78 \%)$, mp. 180-82 ${ }^{\circ} \mathrm{C}$. Anal. Calcd. for $\mathrm{C}_{20} \mathrm{H}_{16} \mathrm{~N}_{4} \mathrm{O}_{2} \mathrm{~S}$ (376.44): C, 63.81; H, 4.28; N, 14.88. Found: C, 63.62; H, 4.30; N, 14.94. IR (KBr, cm $\left.{ }^{-1}\right)$ : 3402 and $3360\left(\mathrm{NH}_{2}\right), 3165(\mathrm{NH}), 1648,1641$ (2 CO); 
${ }^{1} \mathrm{H}$ NMR (400 MHz, DMSO-d6): $\delta$, ppm 3.57 (s, 2H, $\left.\mathrm{CH}_{2}\right), 7.31-7.33$ (m, 10H, Ar-H), 7.42(s, $1 \mathrm{H}$, thiophen-H), 7.45(s, 2H, $\left.\mathrm{NH}_{2}\right), 13.61(\mathrm{~s}, 1 \mathrm{H}, \mathrm{NH})$. $\mathrm{MS}(\mathrm{EI}): \mathrm{m} / \mathrm{z}(\%)=376\left(\mathrm{M}^{+}\right)$.

8-Amino-2-benzyl-1,3-dioxo-4-(phenylhydrazono)-1,2,3,4-tetrahydroisoquinoline-6,7dicarboxylic acid dimethyl ester (7). A mixture of dimethyl acetylenedicarboxylate (10 mmol) and $5 \mathbf{d}(3.76 \mathrm{~g}, 10 \mathrm{mmol})$ in dioxan $(5 \mathrm{ml})$ was irradiated in focused microwave equipment at 250 Watt, $210{ }^{\circ} \mathrm{C}$ for 15 minutes. The reaction mixture was evaporated then washed with ethanol. The solid product, so formed, was collected by filtration and crystallized from dioxan as brown powder; yield $3.21 \mathrm{~g}(66 \%)$, mp. $292-94{ }^{\circ} \mathrm{C}$. Anal. Calcd. for $\mathrm{C}_{26} \mathrm{H}_{22} \mathrm{~N}_{4} \mathrm{O}_{6}$ (486.49): C, 64.19; H, 4.56; N, 11.52. Found: C, 64.23; H, 4.62; N, 11.48. IR (KBr, cm $\left.{ }^{-1}\right): 3373(\mathrm{NH}), 3208$, $3169\left(\mathrm{NH}_{2}\right) 1742,1682,1642,1640$ (4 CO); ${ }^{1} \mathrm{H}$ NMR (400 MHz, DMSO-d6): $\delta$, ppm 3.91 (s, $\left.3 \mathrm{H}, \mathrm{OCH}_{3}\right), 3.97\left(\mathrm{~s}, 3 \mathrm{H}, \mathrm{OCH}_{3}\right), 5.18\left(\mathrm{~s}, 2 \mathrm{H}, \mathrm{NH}_{2}, \mathrm{D}_{2} \mathrm{O}\right.$ exchangeable $), 7.21-7.35(\mathrm{~m}, 11 \mathrm{H}, \mathrm{Ar}-\mathrm{H})$, 13.7(s, 1H, NH). MS (EI): $\mathrm{m} / \mathrm{z}(\%)=486\left(\mathrm{M}^{+}\right)$.

7-Benzyl-6,8-dioxo-2-phenyl-2,6,7,8-tetrahydropyrido[3,4-c]pyridazine-5-carbonitrile (9).

Method A. A solution of $\mathbf{4 d}(10 \mathrm{mmol})$ and DMFDMA (10 mmol) was irradiated in a focused microwave at $150 \mathrm{Watt}, 180^{\circ} \mathrm{C}$ for 5 minutes. The solid product obtained was crystallized from ethanol/dioxan, to give yellow crystals were formed.

Method B. A solution of $\mathbf{4 d}(10 \mathrm{mmol})$ and DMFDMA $(10 \mathrm{mmol})$ was refluxed in xylene for $18 \mathrm{hr}$. The solid product obtained was crystallized from ethanol / dioxan as yellow crystals.

Yield 2.73 g (77\%), mp. 267-69 ${ }^{\circ} \mathrm{C}$. Anal. Calcd. for $\mathrm{C}_{21} \mathrm{H}_{14} \mathrm{~N}_{4} \mathrm{O}_{2}$ (354.37): C, 71.18; H, 3.98; N, 15.81. Found: C, 71.48; H, 3.99; N, 15.97. IR (KBr, cm $\left.{ }^{-1}\right): 2208(\mathrm{CN}), 1681,1645(\mathrm{CO})$; ${ }^{1} \mathrm{H}$ NMR (400 MHz, DMSO-d6): $\delta$, ppm 5.11(s, 2H, $\left.\mathrm{CH}_{2}\right), 7.24-7.32(\mathrm{~m}, 5 \mathrm{H}, \mathrm{Ar}-\mathrm{H}), 7.48(\mathrm{~d}, 1 \mathrm{H}$, pyridazine $\mathrm{H}, J=8 \mathrm{~Hz}), 7.86(\mathrm{~m}, 5 \mathrm{H}, \mathrm{Ar}-\mathrm{H}), 9.05(\mathrm{~s}, 1 \mathrm{H}$, pyridazine $\mathrm{H}, \mathrm{J}=8 \mathrm{~Hz}) ;{ }^{13} \mathrm{C}$ NMR $(100$ MHz, DMSO-d6): $\delta$, ppm 44.1, 78, 117.1, 118.2, 122.7, 128.1, 128.6, 129.3, 130.8, 131.0, 137.9, $138.9,140.6,140.7,143.8,159.0,162.3$. MS (EI): $\mathrm{m} / \mathrm{z}(\%)=354\left(\mathrm{M}^{+}\right)$.

\section{Acknowledgements}

The authors are grateful to Kuwait University research administration for the financial support of project SC11/02 and for SAF facilities project GC01/02 and Gs03/01.

\section{References}

1. Elnagdi M. H.; Negm A. M.; Erian A. W. Liebigs Ann. Chem. 1989, 1255.

2. Al-Omran F.; Khalik M. M. A.; Al-Awadhi H.; Elnagdi M. H.; Harb A. F. A.; Metwally S. A. M. Tetrahedron 52, 1992, 11915.

3. Elnagdi M. H.; Erian A. W. Liebigs Ann. Chem. 1990, 1215.

4. Al-Awadhi H.; Al-Omran F.; Infantes L.; Foces-Foces C.; Jagerovic M.; Elguero J.; Elnagdi M. H. Tetrahedron 1995, 51, 12745. 
5. Elnagdi M. H; Negm A. M.; Hassan E. M.; ElBoreiy A. J. Chem. Res. (S) 1993, 130.

6. Abu-Shanab F. A.; Wakefield B.; Al-Omran F.; Abdel Khalik M. M.; Elnagdi M. H. J. Chem. Res. (S) 1995, 488; (M) 1995, 2924.

7. Al-Saleh B. , Hilmy N. M. , El-Apasery M. A. and Elnagdi M. H. J. Heterocyclic Chem. 2006, 43, 1575

8. Al-Saleh B.; Abdelkhalik M. M.; El-Apasery M. A.; Elnagdi M. H. J. Chem. Res. 2005, 23.

9. Sopbué Fondio E.; Döpp D.; Henkel G. Tetrahedron 2006, 62, 7121.

10. Sopbué Fondio E.; Döpp D. Arkivoc, 2006, (x), 90.

11. Bellow K. A. Dyes and Pigments 1995, 28, 83. 